\title{
Theorical analysis of a monolithic all-active three-section semiconductor laser
}

\author{
Mohammed Mehdi Bouchene, ${ }^{* 1}$ Rachid Hamdi, ${ }^{1}$ and Qin Zou ${ }^{2}$ \\ ${ }^{1}$ Laboratoire des Télécommunications (LT), Faculté des Sciences et de la Technologie, Université 8 mai \\ 1945 Guelma, 24000-Bp.401, Algeria \\ ${ }^{2}$ Institut Mines-Télécom, Télécom SudParis, UMR 5157 CNRS, Université Paris-Saclay, \\ 9 rue Charles Fourier, 91011 Evry Cedex, France
}

Received December 01, 2017; accepted December 30, 2017; published December 31, 2017

\begin{abstract}
We propose a novel semiconductor laser structure. It is composed of three cascaded active sections: a Fabry-Pérot laser section sandwiched between two gain-coupled distributed feedback (DFB) laser sections. We have modeled this multi-section structure. The simulation results show that compared with index- and gain-coupled DFB lasers, a significant reduction in elongitudinal spatial-hole burning can be obtained with the proposed device, and this is what leads to a stable single longitudinal mode operation at relatively high optical power with a SMSR exceeding 56dB.
\end{abstract}

Multi-section semiconductor lasers have attracted considerable attention because of their unique properties such as a wide wavelength tuning range [1], high-speed direct modulation [2], and resulting applications. In recent years, various structures of multi-section laser diodes have been proposed based on monolithic cavity configuration [3-4].

In this letter, we propose a multi-section semiconductor laser with a monolithic cavity structure. The schematic representation of the proposed device is shown in Figure 1. The structure is considered to be a cascade of all-active three-section semiconductor lasers: two DFB lasers with uniform grating and with no-phase shifts at rear and front facets sections and a Fabry-Pérot laser in the middle. All the three sections have the same material and structural parameters (see Table 1) with an equal length and also biased with symmetrical driving current. We name this device as the monolithic three active-section (M3-As) laser.

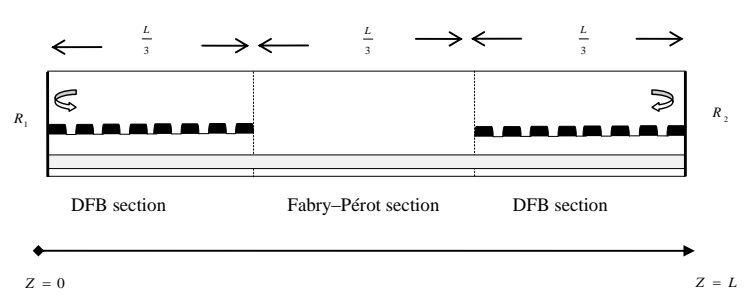

Fig. 1. Schematic representation of a M3-As semiconductor laser.

E-mail: bouchenemahdi@gmail.com

http://www.photonics.pl/PLP
Table 1: Structural and material parameters.

\begin{tabular}{|c|c|c|c|}
\hline Parameter & Value & Description & Unit \\
\hline$B_{s p o n t}$ & 1 & Bimolecular coefficient & $10^{-10} \mathrm{~cm}^{3} / \mathrm{s}$ \\
\hline$C_{\text {Aug }}$ & 3 & Auger coefficient & $10^{-29} \mathrm{~cm}^{6} / \mathrm{s}$ \\
\hline$G_{N}$ & 3 & Differential field gain & $10^{-16} \mathrm{~cm}^{2}$ \\
\hline$\alpha_{m}$ & 40 & Waveguide loss & $\mathrm{cm}^{-1}$ \\
\hline$N_{0}$ & 1.5 & Transparency carrier density & $10^{18} \mathrm{~cm}^{3}$ \\
\hline$n_{\text {eff }}{ }^{\circ}$ & 3.27 & Effective phase refractive index & \\
\hline$n_{g}$ & 3.7 & Effective group refractive index & \\
\hline$L$ & 400 & Total cavity length & $\mu \mathrm{m}$ \\
\hline$w$ & 3.5 & Active layer width & $\mu \mathrm{m}$ \\
\hline$d$ & 0.47 & Active layer thickness & $\mu \mathrm{m}$ \\
\hline$\Gamma$ & 0.35 & Confinement factor & \\
\hline$R_{2}, \mathrm{R}_{1}$ & 0.32 & $\begin{array}{l}\text { Right and left facet power } \\
\text { reflectivity }\end{array}$ & \\
\hline$\lambda_{0}$ & 1550 & Wavelength & $\mathrm{nm}$ \\
\hline$\alpha_{H}$ & 4.86 & Linewidth enhancement factor & \\
\hline$\varepsilon$ & 1 & Gain compression factor & $10^{-17} \mathrm{~cm}^{3}$ \\
\hline$\beta$ & $10^{-4}$ & $\begin{array}{c}\text { Spontaneous emission } \\
\text { coefficient }\end{array}$ & \\
\hline
\end{tabular}

The theoretical framework used in our simulation is the one-dimensional Time Domain Travelling Wave (TDTW) model. The time-dependent coupled wave equations for the analysis different types of semiconductor lasers are expressed as follows [5]:

$$
\begin{aligned}
& \frac{1}{C_{G}} \frac{\partial E^{+}}{\partial t}+\frac{\partial E^{+}}{\partial z}=\left(\frac{\Gamma G_{N}\left\{N-N_{0}\right\}}{2(1+\varepsilon S)}-\frac{\alpha m}{2}-\mathrm{j} \delta\right) E^{+}+ \\
& j \kappa E^{-}+\xi_{+} \\
& \frac{1}{C_{G}} \frac{\partial E^{-}}{\partial t}-\frac{\partial E^{-}}{\partial z}=\left(\frac{\Gamma G_{N}\left\{N-N_{0}\right\}}{2(1+\varepsilon S)}-\frac{\alpha m}{2}-\mathrm{j} \delta\right) E^{-}+ \\
& j \kappa E^{+}+\xi_{-}
\end{aligned}
$$

In the above equations, $E^{+}(z, t), E^{-}(z, t)$ are respectively the forward and backward slowly varying amplitudes of (C) 2017 Photonics Society of Poland 
the traveling optical fields, and $\kappa$ is the coupling coefficient between them. For a mixed-coupled DFB laser [6]: $\kappa=\kappa_{i}+j \kappa_{g}$, where $\kappa_{i}$ is the index-coupling coefficient, and $\kappa_{g}$ is the gain-coupling coefficient. For a purely index-coupled DFB laser, $\kappa_{g}=0$, and for a purely gain-coupled DFB laser, $\kappa_{i}=0$, and for a Fabry-Pérot laser, $\kappa=0 . S$ is the photon density, $\alpha m$ is the material loss, $\Gamma$ is the confinement factor, $\delta$ is the detuning factor defined as:

$$
\delta(z, t)=\frac{2 \pi}{\lambda_{0}} n_{e f f}(z, t)-\frac{\pi}{\Lambda},
$$

$n_{\text {eff }}$ is the effective refractive index, which is given by:

$$
n_{e f f}(z, t)=n_{e f f}{ }^{0}-\Gamma \alpha_{m}\left(\frac{\Gamma G_{N}\left\{N(z, t)-N_{0}\right\}}{2(1+\varepsilon S(z, t))}\right) \frac{\lambda_{0}}{4 \pi},
$$

$\xi_{+}(z, \mathrm{t})$ and $\xi_{-}(z, \mathrm{t})$ are the spontaneous emission noises which are modeled in calculation by the Gaussian random number generator [7]. The rate equation for the carrier density $N$ is given by [8]:

$$
\frac{\partial N}{\partial t}=\frac{J(t)}{e d}-\frac{N}{\tau_{s}}-B_{\text {Spont }} N^{2}-C_{A u g} N^{3}-G C_{G} S
$$

where $J(t)$ is the injection current, $e$ is the modulus of the electron charge, $d$ is the volume of the active layer, $\tau_{s}$ is the carrier life time, $B_{\text {spont }}$ is the radiative spontaneous emission coefficient, $C_{A u g}$ is the Auger recombination coefficient. The boundary conditions are defined at the rear and front facets of the laser as follows:

$$
\begin{aligned}
& E^{+}((z=0), t)=\sqrt{R_{1}} E^{-}(z=0, t) \\
& E^{-}(z=L, t)=\sqrt{R_{2}} E^{+}(z=L, t)
\end{aligned}
$$

$R_{1}, R_{2}$ are the left and right facet power reflectivities. The TDTW model is solved explicitly in the time domain by using the Finite-Difference Time-Domain (FDTD) method [9]. The DFB lasers used in the M3-As configuration are gain-coupled grating because they show important advantages in terms of modal phase resonance as demonstrated in Fig. 2.

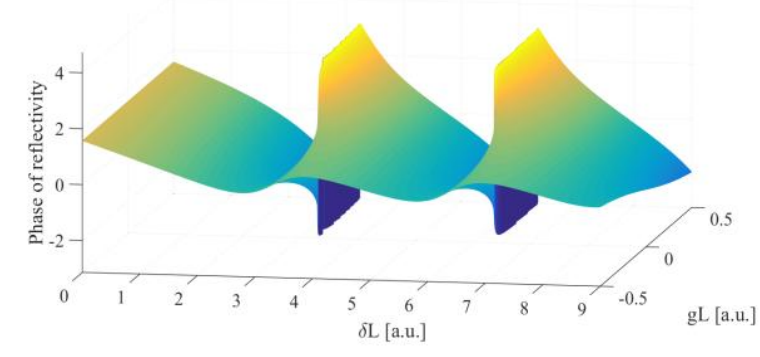

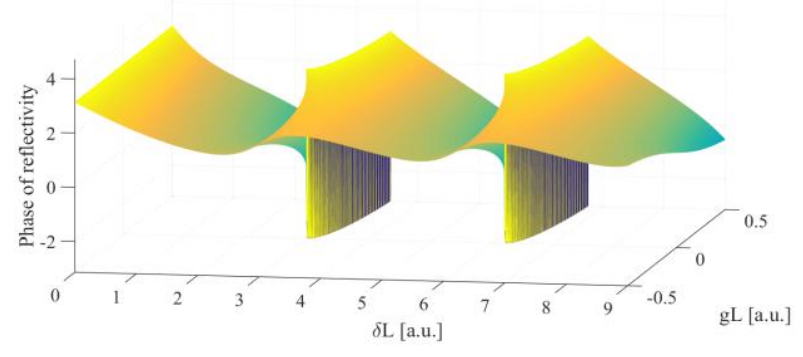

Fig. 2. Optical-field phase reflectivity in index and gain Bragg gratings.

Figure 3 shows the $\mathrm{CW}$ spectrum of an index-coupled DFB laser with $\kappa L=2$ and a gain-coupled DFB laser and a M-3As laser biased at $51.15 \mathrm{~mA}$. The emitted power from each facet for the three devices is respectively: $2.4830 \mathrm{~mW}, 4.3650 \mathrm{~mW}$, and $5.0556 \mathrm{~mW}$. The spectrum is obtained by applying the Fourier transform on the time samples of an optical field calculated by the TDTW model after the devices reach a steady-state condition [10]. It can be observed that the M3-As laser has the worst side-mode suppression ratio (SMSR). These results suggest that the M3-As laser is not suitable in a low output power range whereas the conventional DFB lasers show better performance.
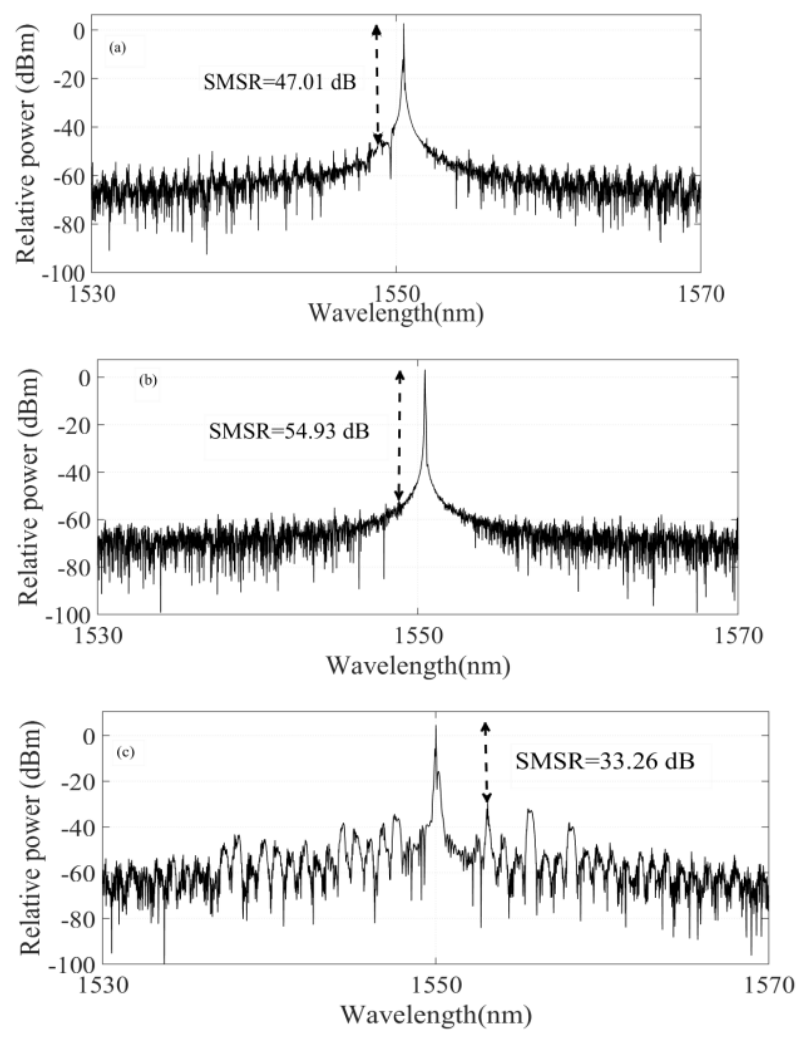

Fig. 3. CW optical spectra of: (a) an index-coupled DFB laser ( $\kappa L=2$ ), (b) a gain-coupled DFB laser, (c) a M3-As laser. 
Figure 4 shows the $\mathrm{CW}$ spectra of an index-coupled $(\kappa L=2$ ) laser, a gain-coupled DFB laser, and a M-3As laser biased at $250.78 \mathrm{~mA}$. The average power emitted from each facet for the three devices is respectively $25.4032 \mathrm{~mW}, 34.9140 \mathrm{~mW}$, and $37.2348 \mathrm{~mW}$. It can be seen that both index- and gain-coupled DFB lasers have similar performance under strong injection current where the spectral linewidth has been drastically broaden and the laser exhibits a multimode operation as a result of strong longitudinal spatial-hole burning in a conventional DFB laser due to the modulation of the active layer refractive index caused by the change of carrier density which produces a dynamic frequency chirp in laser oscillation [11-12]. In contrary, the M3-As laser generates a single frequency oscillation and excellent wavelength stability at high output power with an improved side mode suppression ration equal to $56.08 \mathrm{~dB}$.
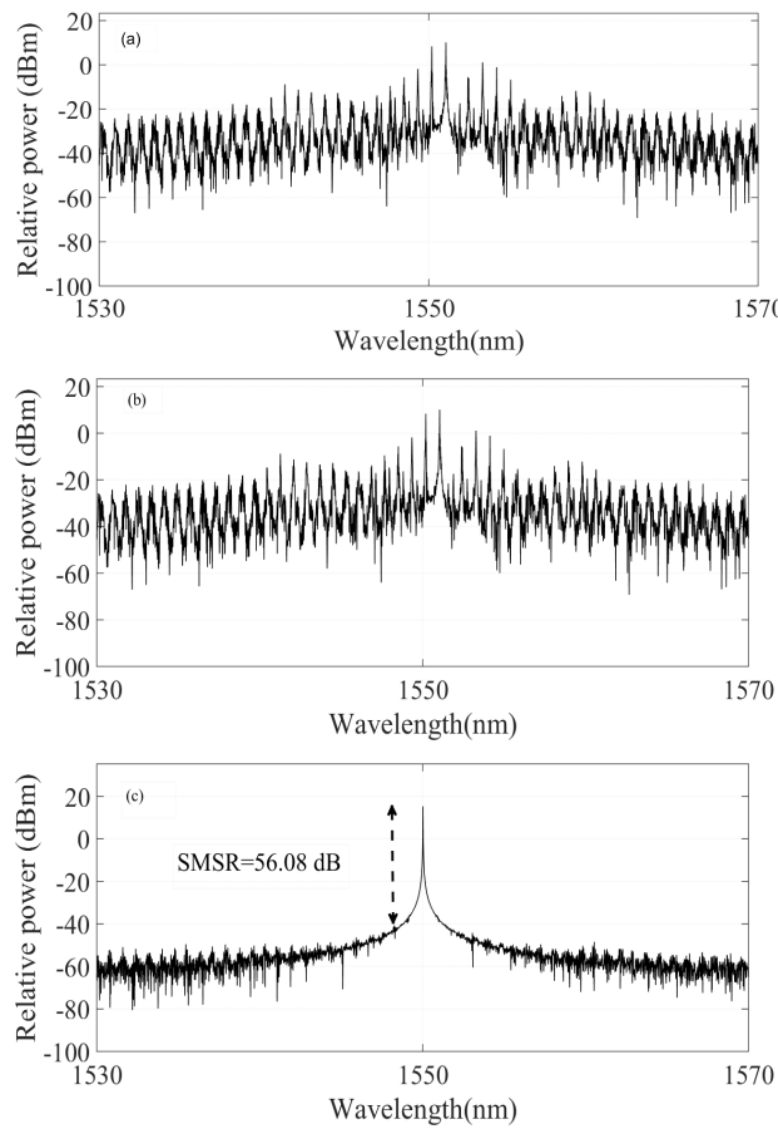

Fig. 4. CW optical spectra of: (a) an index-coupled DFB laser ( $\kappa L=2$ ), (b) a gain-coupled DFB laser, (c) a M3-As laser.

In order to confirm these results, we plot in Figure 5 the internal spatial distribution of optical intensity inside the laser (cavity) structure. As can be seen in the figure, the optical intensity in index- and gain-coupled DFB lasers builds up to achieve the maximum around the centre of the laser. Therefore, strong longitudinal spatial-hole burning occurs as the electron density decreases with the increase of the refractive index of the active region. On the other hand, it is clear that the M3-As laser has a near flat optical intensity profile; and this uniformity of optical field distribution is achieved by the uncorrugated section which represents the F-P laser section that creates a longitudinal field stop-band profile. This effect results in a significant reduction of longitudinal spatial-hole burning.

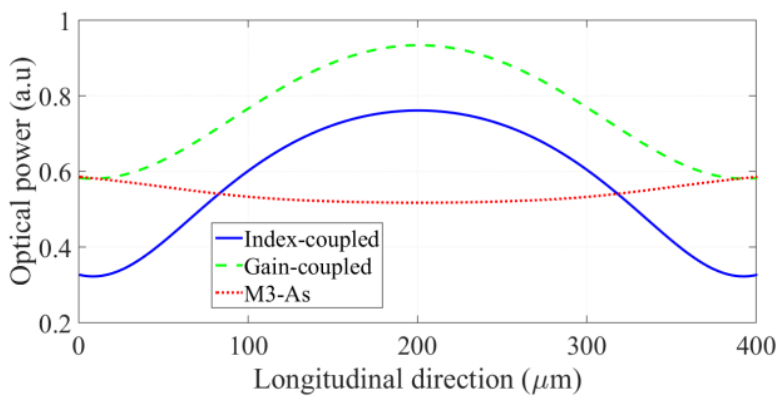

Fig. 5. Internal optical power distribution along the longitudinal direction of a M3-As laser.

In summary, we have investigated theoretically the novel structure of a monolithic multi-section semiconductor laser operating around a wavelength of $1550 \mathrm{~nm}$. Single-frequency operation for a relatively high output power emission has been successfully achieved. The results suggest that the M3-As laser could be used in applications that require a high output power narrowlinewidth.

\section{References}

[1] L.A. Coldren, IEEE J. Select. Topics Quant. Electron. 6, 988 (2000).

[2] O. Kjebon, R. Schatz, S. Lourdudoss, S. Nilsson, B. Stalnacke, L. Backbom, Electron. Lett. 33(6), 488 (1997).

[3] N. Kim, J. Shin, E. Sim, C.W. Lee, D.-S. Yee, M.Y. Jeon, Y. Jang, K.H. Park, Opt. Expr. 17(16), 13851 (2009).

[4] M.J. Wallace, R. ORreilly Meehan, R.R Enright, F. Bello, D. Mccloskey, B. Barabadi, E.N. Wang, J.F. Donegan, Opt. Expr. 25(13), 14426 (2017).

[5] J.E. Carroll, J.E.A. Whiteaway, R.G.S. Plumb, Distributed feedback semiconductor lasers (IEE and SPIE, 1998).

[6] H. Ghafour-Shiraz, Distributed Feedback Laser Diodes and Optical Tunable Filters (Wiley, 2003).

[7] D.D. Marcenac, Ph.D dissertation (University of Cambridge, 1993).

[8] L.M. Zhang, J.E. Carroll, C. Tsang, IEEE J. Quant. Electr. 29, 1722 (1993).

[9] W. Li, W.-P. Huang, X. Li, J. Hong, IEEE J. Quant. Electro. 36(10), 1110 (2000).

[10] B.M. Mehdi, H. Rachid, in Proc. 3rd Intern. Conf. on Embedded Systems in Telecomm. and Instrument., Annaba, Algeria (2016).

[11] C. Henry, IEEE J.Quant. Electr. QE-18, 259 (1982).

[12] K. Takaki, T. Kise, K. Maruyama, N. Yamanaka, M. Funabashi, A. Kasukawa, IEEE J. Quant. Electr. 39, 1060 (2003). 